\title{
Use of a Multiplex PCR for the Detection of Toxin-Encoding Genes netB and tpeL in Strains of Clostridium perfringens
}

\author{
Matthew A. Bailey, Kenneth S. Macklin, and James T. Krehling \\ Department of Poultry Science, Auburn University, 201 Poultry Science Building, Auburn, AL 36849-5416, USA \\ Correspondence should be addressed to Kenneth S. Macklin; macklks@auburn.edu
}

Received 8 October 2013; Accepted 13 November 2013

Academic Editors: A. Balkema-Buschmann and A. Fernandez

Copyright (C) 2013 Matthew A. Bailey et al. This is an open access article distributed under the Creative Commons Attribution License, which permits unrestricted use, distribution, and reproduction in any medium, provided the original work is properly cited.

\begin{abstract}
Some studies have shown that the NetB toxin may be an important virulence factor of Clostridium perfringens associated necrotic enteritis in poultry. Additionally, research has shown that strains of $C$. perfringens positive for both the netB gene and a second toxin-encoding gene, tpeL, appear to be more virulent than strains with only net $B$. In the past, detection of these genes has been performed relatively inefficiently using two single locus PCRs. This report describes a novel multiplex PCR developed to detect netB and tpeL simultaneously in C. perfringens strains isolated from cases of necrotic enteritis in broilers, providing a more efficient diagnostic tool in the screening of strains for these genes.
\end{abstract}

\section{Introduction}

The disease necrotic enteritis (NE) is a major issue affecting the poultry industry, causing extensive economic loss through mortality, reduced bird performance, and carcass condemnation at slaughter $[1,2]$. Caused by the bacterium Clostridium perfringens (CP), NE is characterized by necrotic lesions, primarily in the jejunum and ileum, which can vary in severity from thickened mucosa and multifocal ulceration in less severe cases to the formation of a greenish or yellowish pseudomembrane in the case of extensive mucosa inflammation and necrosis [3]. In the past, alpha-toxin produced by $\mathrm{CP}$ type $\mathrm{A}$ has been implicated as the primary virulence factor in NE pathogenesis $[2,4,5]$; however, the NetB toxin, encoded by the net $B$ gene, was shown to be important for the virulence of certain strains $[6,7]$. Another toxin, TpeL (encoded by the tpeL gene) [8], is a potential virulence factor as well. In a recent study, inoculation of broilers with strains positive for both tpeL and $n e t B$ was associated with greater severity of gross lesions over strains with only netB [9].

Existing studies ascertaining the prevalence of net $B$ and tpeL have been limited to certain geographic populations of CP, mainly in Australia, Belgium, Denmark, Sweden, and Canada $[1,7]$. In the United States, studies on the prevalence of these genes have analyzed CP populations from New
England, New York, and Pennsylvania [10]. The relatively small number of sampled CP populations underlines a need for more analysis to determine the importance of these genes on a worldwide scale. Additionally, detection of these genes has been performed relatively inefficiently using two single locus PCRs. This report describes a multiplex PCR developed to detect $n e t B$ and tpeL simultaneously, providing a more efficient tool in screening CP strains for these genes.

\section{Materials and Methods}

Strains used as positive controls were provided by J.G. Songer of Iowa State University (JGS-1870 and JGS-4140) and the Mitchem-Sparks Regional Diagnostic Laboratory located in Boaz, Alabama (C103-99). All strains (Table 1) were prepared for DNA extraction by enriching in cooked meat medium (HiMedia Laboratories Pvt. Ltd., Mumbai, India) for $24 \mathrm{hr}$ and then streaking the enriched samples for isolation onto trypticase soy agar with 5\% sheep's blood (BD Diagnostics, Franklin Lakes, NJ). After $24-48 \mathrm{hr}$, a single colony exhibiting typical hemolysis was placed into brain-heart infusion (BHI) broth (BD Diagnostics) and incubated for $24 \mathrm{hr}$. All incubation was carried out at $37^{\circ} \mathrm{C}$ within a Bactron IV Anaerobic Environmental Chamber (Shel Lab, Cornelius, OR) with the following atmospheric conditions: $90 \% \mathrm{~N}_{2}, 5 \% \mathrm{CO}_{2}$, and $5 \%$ 
TABLE 1: Bacterial strains used as positive and negative controls in a multiplex polymerase chain reaction designed for detection of Clostridium perfringens genes netB and tpeL.

\begin{tabular}{lccccc}
\hline Strain ID number & Species & netB & tpeL & Relevance & Source \\
\hline C103-99 & Clostridium perfringens & + & + & Positive control & Necrotic enteritis \\
JGS-1870 & Clostridium perfringens & + & + & Positive control & Necrotic enteritis \\
JGS-4140 & Clostridium perfringens & + & + & Positive control & Necrotic enteritis \\
$232-2$ & Clostridium bifermentans & - & - & Negative control & Poultry litter \\
$259-14$ & Bacteroides caccae & - & - & Negative control & Poultry litter \\
$250-4$ & Wolinella sp. & - & - & Negative control & Poultry litter \\
\hline
\end{tabular}

TABLE 2: Specifications of primers used in a multiplex PCR for the detection of netB and tpeL.

\begin{tabular}{|c|c|c|c|}
\hline Primer ID & Sequence & Target gene & Product length \\
\hline netB5F & CGCTTCACATAAAGGTTGGAAGGC & \multirow{2}{*}{$n e t B$} & \multirow{2}{*}{316} \\
\hline netB5R & TCCAGCACCAGCAGTTTTTCCT & & \\
\hline AKP80* & ATATAGAGTCAAGCAGTGGAG & \multirow{2}{*}{ tpeL } & \multirow{2}{*}{466} \\
\hline AKP81* & GGAATACCACTTGATATACCTG & & \\
\hline
\end{tabular}

* Primers previously published by Keyburn et al., 2010 [7].

$\mathrm{H}_{2}$. After incubation, BHI broth cultures were centrifuged at $16,000 \mathrm{~g}$ for $5 \mathrm{~min}$ and the broth supernatant was aspirated and discarded. Utilizing a Wizard Genomic DNA Purification Kit (Promega Corp., Madison, WI), DNA was extracted from the resulting bacterial pellet. Extracted DNA was analyzed for quantity and purity with a Nanodrop 1000 Spectrophotometer (Thermo Scientific Inc., Bremen, Germany) according to the manufacturer's instructions. The standard for DNA purity was a $260 / 280 \mathrm{~nm}$ absorbance ratio of 1.8-2.0.

A previously published primer set [7] was used for the detection of tpeL and a primer set detecting netB was designed using the primer Basic Local Alignment Search Tool (BLAST) function on the National Center for Biotechnology website. Primer specifications are listed in Table 2. The net $B$ primers were confirmed to target mostly conserved sequences by performing a sequence alignment with 47 netB sequences in GenBank using genetic analysis software.

EconoTaq PLUS GREEN Master Mix (Lucigen Corp., Middleton, WI) was used at $1 \mathrm{x}$ concentration providing Taq polymerase, $\mathrm{MgCl}_{2}$, dNTPs, and reaction buffer ( $\mathrm{pH} 9.0$ ). Reagent concentrations of the optimized multiplex were as follows: 1.2 units/25 uL of Taq DNA polymerase; $1.44 \mathrm{mM}$ of $\mathrm{MgCl}_{2} ; 192 \mathrm{uM}$ of each dNTP; $0.2 \mathrm{uM}$ of each primer; and $100 \mathrm{ng} / 25 \mathrm{uL}$ of template DNA. The following cycling conditions were used: an initial denaturing step at $95^{\circ} \mathrm{C}$ for $5 \mathrm{~min} ; 40$ cycles of denaturing at $95^{\circ} \mathrm{C}$ for $30 \mathrm{sec}$; annealing at $55^{\circ} \mathrm{C}$ for $30 \mathrm{sec}$, and extension at $72^{\circ} \mathrm{C}$ for $30 \mathrm{sec}$, with a final extension step at $72^{\circ} \mathrm{C}$ for $7 \mathrm{~min}$.

Products were separated by electrophoresis in a $2 \%$ agarose gel. Gels and running buffer were made using $1 x$ concentrations of AccuGENE TBE buffer (Lonza Group, Basel, Switzerland). For visualization of bands, $1 \mathrm{uL}$ of an ethidium bromide solution $(10 \mathrm{mg} / \mathrm{mL})$ was added to the molten gel before solidifying. Electrophoresis took place at $100 \mathrm{v}$ for about one hr or until sufficient separation between products occurred. A $100 \mathrm{bp}$ DNA Ladder (Promega) was utilized as a DNA size standard. Bands corresponding to the expected amplicons were excised and DNA was purified using a GFX PCR DNA and Gel Band Purification Kit (GE Healthcare, Buckinghamshire, UK).

Forward and reverse strands of the purified DNA were sequenced by Lucigen and a consensus sequence was determined for each amplicon. These consensus sequences were then subjected to a nucleotide BLAST search using the NCBI database Nucleotide collection (nr/nt) to calculate the likelihood that each PCR product was produced from the target gene.

\section{Results}

After the analysis of 47 netB gene sequences published in GenBank, the target sequences for both forward (netB5F) and reverse (netB5R) primers proved to be mostly conserved. A single polymorphism was present at site number 756 of the alignment, with $21(44.7 \%)$ of the sequences having $\mathrm{G}$ at this site and 26 (55.3\%) having A. This site was complementary to the sixth base from the $5^{\prime}$ end of primer netB5R and resulted in a single A:C mismatch between the primer and 55.3\% of the gene sequences. This same mutation affects primers previously described by Keyburn et al. [6], resulting in a G:T mismatch between the seventh base from the $5^{\prime}$ end of AKP78 (the forward primer) and $24(51.1 \%)$ of the analyzed gene sequences.

Gel electrophoresis of PCR products confirmed the amplification of the target sequences for all three positive controls. Bands of the expected size corresponding to the $n e t B(316 \mathrm{bp})$ and tpeL (466 bp) fragments were observed for the positive controls, and no bands were observed for the negative controls. Sequences obtained from PCR products matched the target sequence (at least 99\% max identity) with significantly low $E$-values after performing a BLAST search (Table 3).

\section{Discussion}

The multiplex PCR described in this report successfully detected the netB and tpeL genes in three control organisms 
TABLE 3: BLAST output for sequenced PCR products*.

\begin{tabular}{|c|c|c|c|c|c|}
\hline $\begin{array}{l}\text { Target } \\
\text { gene }\end{array}$ & $\begin{array}{c}\text { Sequence } \\
\text { source }^{\dagger}\end{array}$ & Description of top result & GenBank accession & Max identity (\%) & $E$-value \\
\hline netB & C103-99 & $\begin{array}{l}\text { Clostridium perfringens strain CP4 plasmid pCP4netB } \\
\text { pathogenicity locus } 1 \text { genomic sequence }\end{array}$ & JF837812.1 & 100 & $5 \times 10^{-158}$ \\
\hline netB & JGS-1870 & $\begin{array}{l}\text { Clostridium perfringens strain CP4 plasmid pCP4netB } \\
\text { pathogenicity locus } 1 \text { genomic sequence }\end{array}$ & JF837812.1 & 100 & $3 \times 10^{-152}$ \\
\hline netB & JGS-4140 & $\begin{array}{l}\text { Clostridium perfringens strain CP4 plasmid pCP4netB } \\
\text { pathogenicity locus } 1 \text { genomic sequence }\end{array}$ & JF837812.1 & 100 & $1 \times 10^{-144}$ \\
\hline tpeL & C103-99 & $\begin{array}{l}\text { Clostridium perfringens A strain CP4 TpeL (tpeL) gene, } \\
\text { complete cds }\end{array}$ & EU848493.1 & 99 & 0 \\
\hline tpeL & JGS-1870 & $\begin{array}{l}\text { Clostridium perfringens A strain CP4 TpeL (tpeL) gene, } \\
\text { complete cds }\end{array}$ & EU848493.1 & 99 & 0 \\
\hline tpeL & JGS-4140 & $\begin{array}{l}\text { Clostridium perfringens A strain CP4 TpeL (tpeL) gene, } \\
\text { complete cds }\end{array}$ & EU848493.1 & 99 & 0 \\
\hline
\end{tabular}

* Each row corresponds to a single sequenced band.

${ }^{\dagger}$ lists the strain that the sequenced band was amplified from.

and did not produce false positives in the negative controls. The chance of a false negative was minimized by choosing primers that targeted mostly conserved sites within the $C$. perfringens genome. After analysis of 47 sequences, one polymorphism was observed in the target sequence indicating an internal A:C mismatch between the netB5R primer and 55.3\% of the 47 aligned sequences. Although mismatches between a template and the $3^{\prime}$ end of a primer are known to interfere with the active site of DNA polymerase and can result in reduced reaction efficiency [11, 12], internal mismatches, or those closer to the $5^{\prime}$ end, have a much smaller impact [12]. In addition, out of the possible mismatches, $\mathrm{A}: \mathrm{C}$ (purinepyrimidine) mismatches generally produce only minor interferences [12]. Previously published primer AKP7 ${ }^{7}$ contained a G:T mismatch between $51.1 \%$ of the sequences. As G:T is another purine-pyrimidine mismatch and the mismatch also occurred near the $5^{\prime}$ end of the primer, it is comparable to netB5R. In consequence, it is unlikely that this mismatch between the template and netB5R would have a significant impact on product formation. Although evidence suggests the netB and tpeL toxin genes may be important virulence factors for certain strains of $C$. perfringens, relatively few bacterial populations have been screened for these two genes. More populations must be screened to determine the overall impact of these genes in NE. By pairing the detection of both netB and tpeL into one PCR reaction, this multiplex PCR has the potential to increase the efficiency with which strains are screened for these two genes of interest. The effectiveness of this assay has been shown in three control organisms and must now be confirmed using a larger number of strains.

\section{Acknowledgments}

The authors recognize and appreciate the advice of Dr. Mark Liles regarding sequencing and primer analysis; Dr. Joseph Giambrone for the use of his laboratory equipment; and Dr. J. G. Songer and Janice Seibel for providing positive control strains. The authors declare that there is no conflict of interests regarding the publication of this paper.

\section{References}

[1] A. Johansson, A. Aspán, M. Kaldhusdal, and B. E. Engström, "Genetic diversity and prevalence of netB in Clostridium perfringens isolated from a broiler flock affected by mild necrotic enteritis," Veterinary Microbiology, vol. 144, no. 1-2, pp. 87-92, 2010.

[2] F. Van Immerseel, J. I. Rood, R. J. Moore, and R. W. Titball, "Rethinking our understanding of the pathogenesis of necrotic enteritis in chickens," Trends in Microbiology, vol. 17, no. 1, pp. 32-36, 2009.

[3] M. D. Ficken and D. P. Wages, "Necrotic enteritis," in Diseases of Poultry, B. W. Calnek, H. J. Barnes, C. W. Beard, L. R. McDougald, and Y. M. Saif, Eds., pp. 261-264, Iowa State University Press, Ames, Iowa, USA, 10th edition, 1997.

[4] F. Al-Sheikhly and R. B. Truscott, "The pathology of necrotic enteritis of chickens following infusion of crude toxins of Clostridium perfringens into the duodenum," Avian Diseases, vol. 21, no. 2, pp. 241-255, 1977.

[5] A. L. Keyburn, S. A. Sheedy, M. E. Ford et al., "Alpha-toxin of Clostridium perfringens is not an essential virulence factor in necrotic enteritis in chickens," Infection and Immunity, vol. 74, no. 11, pp. 6496-6500, 2006.

[6] A. L. Keyburn, J. D. Boyce, P. Vaz et al., "netB, a new toxin that is associated with avian necrotic enteritis caused by Clostridium perfringens," PLoS Pathogens, vol. 4, article e26, 2008.

[7] A. L. Keyburn, X.-X. Yan, T. L. Bannam, F. Van Immerseel, J. I. Rood, and R. J. Moore, "Association between avian necrotic enteritis and Clostridium perfringens strains expressing netB toxin," Veterinary research, vol. 41, article 21, 2010.

[8] K. Amimoto, T. Noro, E. Oishi, and M. Shimizu, "A novel toxin homologous to large clostridial cytotoxins found in culture supernatant of Clostridium perfringens type C," Microbiology, vol. 153, no. 4, pp. 1198-1206, 2007.

[9] C. F. Coursodon, R. D. Glock, K. L. Moore, K. K. Cooper, and J. G. Songer, "TpeL-producing strains of Clostridium perfringens type A are highly virulent for broiler chicks," Anaerobe, vol. 18, no. 1, pp. 117-121, 2012.

[10] T. G. Martin and J. A. Smyth, "Prevalence of netB among some clinical isolates of Clostridium perfringens from animals in the 
United States," Veterinary Microbiology, vol. 136, no. 1-2, pp. 202-205, 2009.

[11] S. Kwok, D. E. Kellogg, N. McKinney et al., "Effects of primertemplate mismatches on the polymerase chain reaction: human immunodeficiency virus type 1 model studies," Nucleic Acids Research, vol. 18, no. 4, pp. 999-1005, 1990.

[12] R. Stadhouders, S. D. Pas, J. Anber, J. Voermans, T. H. M. Mes, and M. Schutten, "The effect of primer-template mismatches on the detection and quantification of nucleic acids using the $5^{\prime}$ nuclease assay," Journal of Molecular Diagnostics, vol. 12, no. 1, pp. 109-117, 2010. 

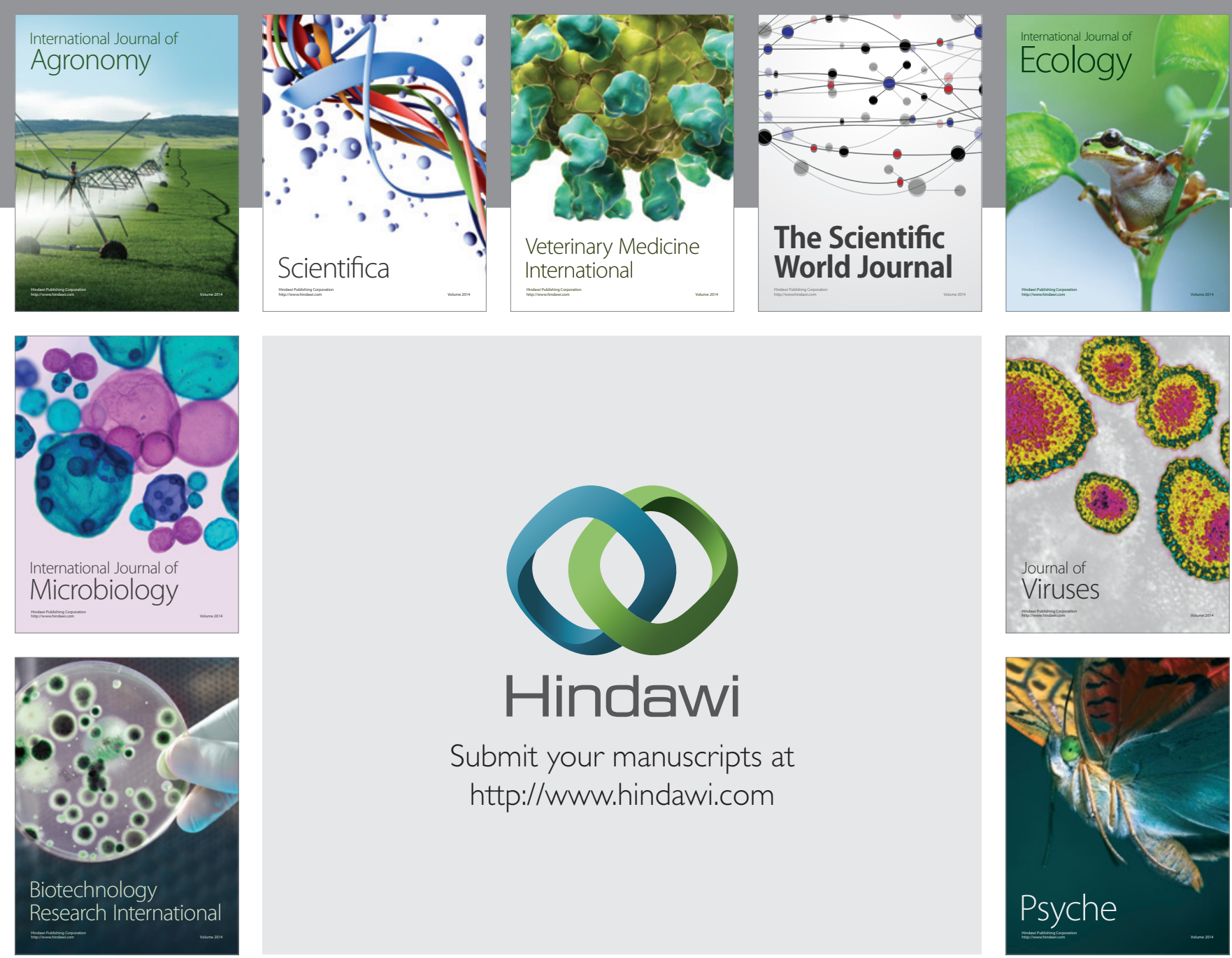

Submit your manuscripts at http://www.hindawi.com
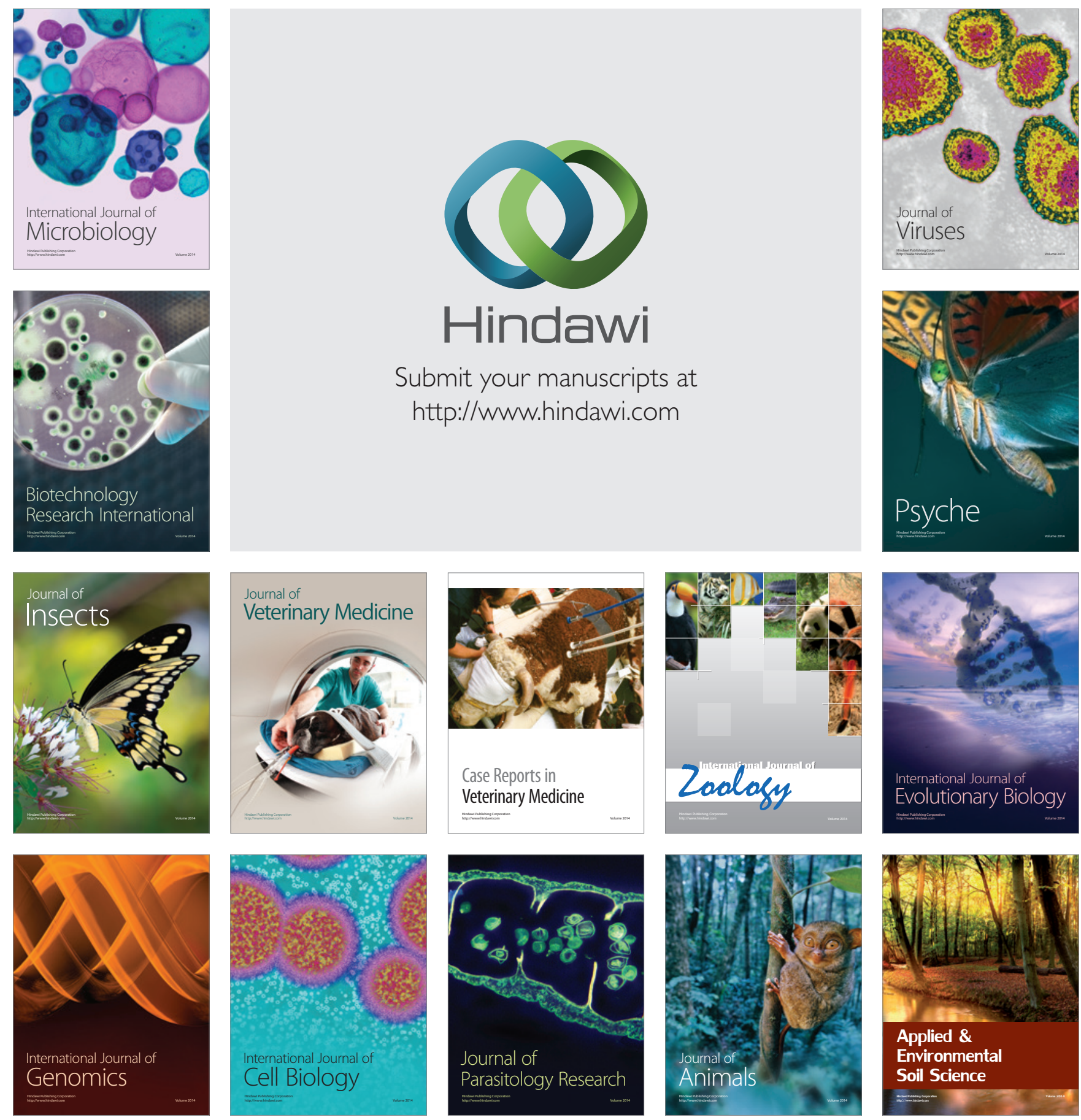